\title{
A Novel Collaboration-Oriented Requirement Model Mapping and Classification Method Based on Domain Knowledge for Complex Mechatronic Products
}

\section{Jiguang Yue}

Tongji University College of Electronic and Information

Fuchao Wu (D295035544@qq.com )

Tongji University College of Electronic and Information https://orcid.org/0000-0002-4365-4843

Jun Liu

Shandong Hoteam software Co., Ltd

Ping Wang

Shandong Hoteam software Co., Ltd

\section{Zhaxin Cui}

Tongji University College of Electronic and Information

Hongyu Zou

Tongji University College of Electronic and Information

\section{Original Article}

Keywords: Complex mechatronic products, Requirement model, Mapping and classification, Product lifecycle model management

Posted Date: February 15th, 2021

DOI: https://doi.org/10.21203/rs.3.rs-193068/v1

License: (9) This work is licensed under a Creative Commons Attribution 4.0 International License. Read Full License 


\section{Title page}

\section{A novel collaboration-oriented requirement model mapping and classification method based on domain knowledge for complex mechatronic products}

Jiguang Yue, born in 1961, is currently a professor at School of Electronic and Information Engineering, Tongji University. He received his $\mathrm{Ph} . \mathrm{D}$. degree in fluid transmission and control from Harbin Institute of Technology. His research interests include advanced measurement and control theory research and technology development in the field of electromechanical integration.

E-mail: yuejiguang@tongji.edu.cn

Fuchao Wu, born in 1998, is currently a master candidate at School of Electronic and Information Engineering, Tongji University. His research interests include product lifecycle model management for complex mechatronic products.

Tel: 18907852667; E-mail: 295035544@qq.com

Jun Liu, born in 1980, is currently the president at Shandong Hoteam software Co., Ltd, China. His research interests include intelligent manufacturing and digital design process manufacturing.

E-mail: liujun@hoteamsoft.com

Ping Wang, born in 1975, is currently the managing director at Shandong Hoteam software Co., Ltd, China. Her research interests include intelligent manufacturing, design knowledge and process management.

E-mail: wpdzxx@126.com

Zhexin Cui, born in 1998, is currently a master candidate at School of Electronic and Information Engineering, Tongji University. His research interests include product lifecycle model management for complex mechatronic products.

E-mail: 932529361@qq.com

Hongyu Zou, born in 1997, is currently a master candidate at School of Electronic and Information Engineering, Tongji University. His research interests include groupware technology.

E-mail: 2030754@tongji.edu.cn

\section{Corresponding author: Fuchao Wu E-mail: 295035544@qq.com}




\title{
A novel collaboration-oriented requirement model mapping and classification method based on domain knowledge for complex mechatronic products
}

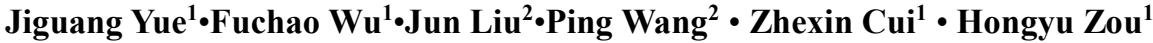

\begin{abstract}
Complex mechatronic products include interdisciplinary, inter-field, and inter-departmental requirement information. Aiming at the low digitization rate of existing product requirement information, a structured and digital product requirement model (RM) is defined. Besides, a collaborationoriented requirement model mapping and classification method for complex mechatronic products is proposed, which realizes the automatic generation of the domain requirement models and the mapping between the requirement models and knowledge graph, thus improving the digitization rate of the product in the requirement phase. A simulated military plane horizontal tail control system is used as a case study to prove the effectiveness and feasibility of this method. Based on Hoteam PLM8.0 platform, a management architecture and the display path of product requirement model are given, which can provide a reference for the research and development of product lifecycle model management (PLMM) system.
\end{abstract}

Keywords: Complex mechatronic products $\bullet$ Requirement model

- Mapping and classification - Product lifecycle model management

$\triangle$ Fuchao Wu

295035544@qq.com

1 Advanced Measurement and Control Technology Laboratory, Tongji University, Shanghai 201804, China

2 Shandong Hoteam software Co., Ltd, Jinan 210000, China

\section{Introduction}

In recent years, the complexity of product research and development is increasing. Therefore, digitalized and networked collaborative manufacturing has become an inevitable trend of enterprise transformation and upgrading [1]. However, for complex mechatronic products, the information in some phases of product lifecycle is still stored and distributed in the form of electronic documents, so it is difficult to realize the efficient management of this information. It is mainly manifested in two aspects:

(1) The information of electronic documents is unstructured. And the digitization rate of this information needs to be improved.
(2) The allocation of inter-disciplinary and interdepartmental product information is mainly done manually. Therefore, product developers spend a lot of time on document sorting and can't focus on creative research and development work.

Product model is equivalent to a small database, which can efficiently query, change and manage [2]. Due to the poor consistency and low communication efficiency of unstructured information in documents, model-based systems engineering (MBSE) has emerged as the times require. Systems Modeling Language (SysML) was widely used by systems engineers [3] and some scholars studied product modeling based on SysML [4-6]. Xu Zihe [7] defined itemized requirement information in Word document and realized the transformation between itemized requirement and SysML requirement diagram. However, SysML is difficult to parse its contents in programming languages. With the development of knowledge engineering and semantic web technology, ontology-based methods [8,9] are also widely used in requirement modeling and analysis. Yaman et al. [10] proposed an ontology framework to represent information and knowledge of engineering product requirements. Based on the framework, they also proposed an ontology model of product design requirement, which provided rich requirement semantic information for requirement storage and analysis. Bakhshandeh et al. [11] proposed a requirement extraction method based on ontology, and they took enterprise ontology and domain ontology as the basic information of requirement extraction. Sheng et al. [12] studied customer requirement modeling and mapping for configuration design, and established a four-tier customer requirement model.

Requirements are the main basis for product research and development, so it must have good traceability. Traceability describes the traceability relationship between system requirement and user requirement [13], which requires that product requirement information must be efficiently retrieved, managed and pushed. As a large-scale knowledge base composed of entities and their relationships, 
knowledge graph $(\mathrm{KG})$ can provide support for efficient search and push of information. The concept of KG was first proposed by Google in 2012. At present, KG is widely used in natural language processing, intelligent question answering system and intelligent recommendation system, etc. $[14,15]$. Research on KG of RM can provide the basis for efficient retrieval, management and push of RM.

At present, many manufacturing enterprises use product lifecycle management (PLM) system to manage the product information. However, PLM is still a management method based on documents and data. There are two challenges for PLM to improve management efficiency and transform into PLMM. One is how to integrate product models, the other is how to effectively manage the product models [16-18]. Bruno et al. [19] proposed a reference ontology that supported PLM. This reference ontology can be used in different fields and can manage a specific product lifecycle by adding new entities, sub-entities and relationships to its structure. Cui et al. [20] put forward a requirement information management model oriented to PLM to realize customer-requirement-centered information management in PLM system. Chabot et al. [21] proposed an approach to realize the knowledge referential by using a generic approach as well as modular and reusable components. Applying this approach on PLM can improve the management efficiency.

The remaining of this paper is arranged as follows. A structured and digital definition of RM is given in section 2 . Section 3 proposes a RM mapping and classification method based on domain knowledge to automatically generate the domain requirement models of the product. In section 4 , a case study of simulated military aircraft horizontal tail control system is given to verifies the effectivity and feasibility of the proposed RM mapping and classification method. Section 5 puts forward a management architecture and the display path of product requirement model. Finally, conclusions are drawn in section 6 .

\section{Structured and digital definition of RM}

The technical agreement documents of the product are generated when the customer signs the contract with the product manufacturer, which cover the product requirement information such as technical data, the uses and function of the system, order information and detailed technical requirements. Currently, technical agreement documents are mostly stored in electronic documents, and most of the information in these documents is unstructured, which is not conducive to store, retrieve and release. In this paper, we obtain the structured and digital product RM by extracting the requirement information in the technical agreement documents and defining it in a structured and digital manner.

Product requirement information contains at least the following types: product name, type description, function requirements, performance requirements, dimensions, weight requirements, price requirement and delivery time.

Definition 1 Attribute the product name, type description, price requirement, and delivery time as attributes of the product requirement model, since they usually contain only a fixed value. Then RM is denoted as

$$
R M\left\langle\text { product_name,type,price, } d_{\text {_time }}\right\rangle
$$

product_name, type, price and d_time represent product name, type description, price requirement and delivery time, respectively.

Definition 2 Consider function requirements, performance requirements, dimensions, and weight requirements as subsets of RM, since they all contain multiple information. Namely,

$$
R M \supseteq F R \bigcup P R \bigcup D R \bigcup W R
$$

FR, PR, DR and WR represent function requirement subset, performance requirement subset, dimension subset and weight requirement subset, respectively. Each of these subsets may contain multiple requirement information, as in the case of FR,

$$
F R=\left\{f r_{1}, f r_{2}, \cdots, f r_{n}\right\}
$$

$f r_{i}, i=1,2, \cdots, n$ represents the single function requirement information of FR.

For complex mechatronic products, each subset may contain the requirement information of machinery, automatic control, electrical and electronics and other domains. Take FR, for example,

$$
F R=\bigcup_{s=1}^{N} F R_{s}
$$

$F R_{s}$ represents the function requirement subset for the sth domain, while $\mathrm{N}$ is the total number of domains that the complex mechatronic product may involve.

Definition 3 Remember RM of a certain domain for complex products as SRM $\langle$ domain $\rangle$. Take automatic control for example,

$$
F R_{s} \cup P R_{s} \cup D R_{s} \cup W R_{s} \subseteq S R M\langle\text { automatic control }\rangle \subset R M
$$

Where $F R_{s}, P R_{s}, D R_{s}$ and $W R_{s}$ respectively represent the function requirement subset, performance requirement subset, dimension subset and weight requirement subset for the automatic control domain requirement model. The other domains are similar. 


\section{RM mapping and classification method based on domain knowledge for complex mechatronic products}

\subsection{Classification of complex mechatronic product RM} In the design process of complex mechatronic products, each design department needs to design the subsystems according to the corresponding requirement information. Currently, most enterprises allocate the requirements by the general department. In this way, the general department spends a lot of time on document sorting, thus extending the time of overall product research and development. To shorten the product development cycle from the requirement phase, a mapping and classification method of RM based on domain knowledge is proposed, which can automatically generate and push the domain requirement models through computer-aided analysis. The architecture of the method is shown in Figure 1.

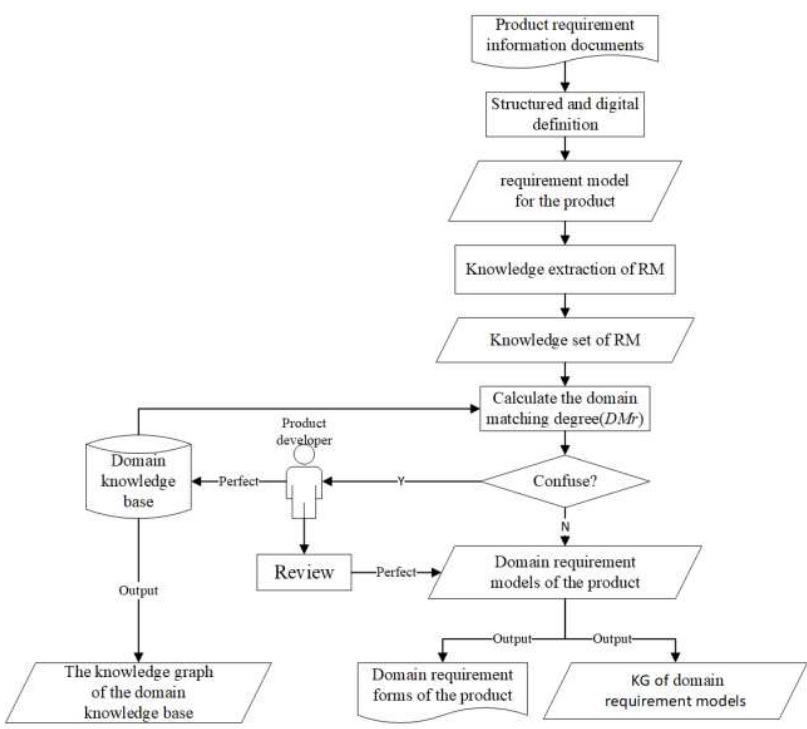

Figure 1 mapping and classification mechanism of RM based on domain knowledge

The main steps of the method are as follows.

Step 1 According to the structured and digital definition, the product requirement model $R M\left\langle\right.$ product_name,type, price, $\left.d_{\text {_time }}\right\rangle$ is obtained.

Step 2 Establishing the domain knowledge bases. When establishing the domain knowledge bases, we should determine the professional category involved in the product and divide a knowledge scope that can clearly express the phenomenon and terms of each domain.

Definition 4 Remember DB as a set of domain knowledge base for all domains involved in the complex mechatronic product,

$$
D B=\bigcup_{s=1}^{N} D B_{s}
$$

Where $D B_{s}$ is the domain knowledge base of the sth domain, and $\mathrm{N}$ is the total number of domains that may be involved in the complex mechatronic product. Each domain knowledge base may contain professional terms, engineering dimensions, units, etc., namely,

$$
D B_{s} \supseteq K N_{s} \bigcup U N_{s} \bigcup E D_{s}
$$

$K N_{s}, U N_{s}, E D_{s}$ represent the professional term set, unit set, and engineering dimension set of the sth domain, respectively.

Step 3 Knowledge retrieval and matching. Obtaining the domain knowledge by searching the domain knowledge bases, so as to calculate the domain matching degree between the requirement information and each domain knowledge base.

Remember $* R$ as a requirement information subset of RM. For a single piece of requirement information $s t r \in * R$.Firstly, we preprocess it, and get

$$
s t r=s t r_{1}+s t r_{2}+\cdots+s t r_{k}
$$

Where $s t r_{j}, j=1,2, \cdots k$ represents the minimum knowledge unit of a single piece of requirement information.

Secondly, the domain knowledge bases are constructed with professional terms, that is

$$
K N_{s}=k n_{s 1}, k n_{s 2}, \cdots, k n_{s m}
$$

Where $k n_{s p}, p=1,2, \cdots, m$ represents one professional term of the sth domain.

Definition 5 The matching relation between the single knowledge $s t r_{j}$ and the single professional term $k r_{s p}$ is the two-valued function $M r s t r_{j}, k n_{s p}$, which is called the meta-matching relation $(\mathrm{Mr})$, then

$$
M r s t r_{j}, k n_{s p}=\left\{\begin{array}{l}
0, s t r_{j} \neq k n_{s p} \\
1, s t r_{j}=k n_{s p}
\end{array}\right.
$$

Definition 6 The matching degree between the single piece of requirement information str and the domain knowledge base of the sth domain is $D M r s t r, s$, which is called the domain matching degree, then 


$$
D M r \text { str, } s=\frac{F M r(s t r, s)}{\sum_{i=1}^{N} F M r(s t r, i)}
$$

Where

$$
F M r(s t r, i)=\sum_{j=1}^{k} \sum_{p=1}^{m} M r s t r_{j}, k n_{i p}
$$

The set of domain matching degrees is DMr, and the maximum value of its element is denoted as $\operatorname{Max}(\mathrm{DMr})$, then

$$
D M r=\bigcup_{s=1}^{N} D M r(s t r, s)
$$

Step 4 According to the calculation results of DMr, the classification result of the requirement information is obtained through certain classification rules.

A single piece of requirement information str may match multiple domains. Denote $\mathrm{S}$ as a set of domains that may match the requirement information str, then

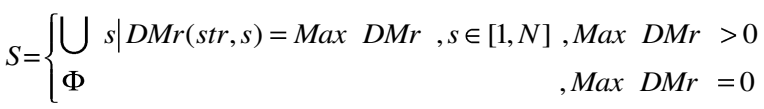

Denote the number of domains in set $\mathrm{S}$ as $\mathrm{c}$. $\mathrm{MH}$ is the confusion set. According to the following classification rules, classify a single piece of requirement information str into the domains that it may belong to.

(1) $\forall s t r \in * R$, if $\mathrm{c}=1$, then $* R_{s}^{i}=* R_{s}^{i-1} \cup\{s t r\}$;

(2) $\forall \operatorname{str} \in * R$, if $\mathrm{c}>1$, then

$$
* R_{s}^{i}=* R_{s}^{i-1} \bigcup\{s t r\}, M H^{i}=M H^{i-1} \bigcup\{s t r\}
$$

(3) $\forall s t r \in * R$, if $\mathrm{c}=0$, then $M H^{i}=M H^{i-1} \cup\{s t r\}$.

Where, $* R_{s}^{i}$ is a requirement information subset of the sth domain requirement model after the addition of requirement information str. $M H^{i}$ is the confusion set after adding requirement information str.

For the second case, str will be classified into multiple domains. For the third case, str cannot be classified into any domain. In both cases, str will be merged into MH. The requirement information in $\mathrm{MH}$ will be output to the product developer. The product developer will further classify the requirement information according to his own professional knowledge and improve the corresponding domain knowledge base.

\subsection{Mapping method between RM and KG}

The product model contains the information of the product. Efficient retrieval, management and reuse of this information is conducive to improving the efficiency of product research and development. In order to support the retrieval, management and reuse of the product model, it is necessary to further condense the information in the product model to form the product knowledge model.

KG contains triples composed of entities, relationships, and attributes, such as entity-relationship-entity, entityattribute-attribute value. KG can clearly describe the relationship between product requirement information, domain requirement model and product requirement model, and provide a basis for the design department of each domain to obtain the corresponding requirement information efficiently. After the automatic generation of domain requirement models, $\mathrm{KG}$ corresponding to $\mathrm{RM}$ (RMKG) is generated through certain mapping rules.

Definition 7 Remember that a piece of requirement information in the product requirement knowledge model is a requirement knowledge block, which contains two features: generalized variable description and generalized variable range.

Generalized variable description contains the description of the requirement information, while generalized variable range contains the relevant requirements indicator values for the product.

Definition $8 \mathrm{RMKG}=(E, R) . E$ represents entities. $R\left(E_{1}, E_{2}\right)$ represents the relation between $E_{1}$ and $E_{2} . E_{1}$ is relation subject, while $E_{2}$ is relation object. Also, under the relationship $R, E_{l}$ is the parent entity of $E_{2}$.

Definition $9 E=(T, P, V)$, where $T$ represents entity type, $P$ and $V$ represent entity attribute and attribute value respectively.

When generating RMKG, two types of entities are generated:

(1) The entities mapped from the set elements, that is, the product requirement information, are called the terminal entity $\left(E_{T}\right)$.

(2) The entities mapped from sets, that is, the product requirement model set and product requirement information subsets, are called non-terminal entities $\left(E_{N T}\right)$.

Define the following mapping rules to generate entities, attributes, attribute values and relationships in RMKG.

(1) $\forall A \subseteq R M, \exists p: A \rightarrow E_{N T}^{A} T, P, V$. Then, $p$ is the mapping from set $A$ to entity $E_{N T}^{A} T, P, V$, and $E_{N T}^{A} T, P, V \quad$ is the corresponding non-terminal entity of set $A$. Where, $T$ inherits the set name; $P$ inherits set attributes; $V$ is the attribute value generated by instantiation.

(2) $\forall a \in R M, \exists q: a \rightarrow E_{T}^{a} T, P, V$. Then, $q$ is the mapping from element $a$ to entity $E_{T}^{a} T, P, V$, and $E_{T}^{a} T, P, V$ is the corresponding terminal entity of element 
$a$.

For the terminal entity $E_{T}^{a} T, P, V$, if $a \in M H$, then $T=$ "product requirement information", otherwise $T$ inherits the type of its parent entity. $P$ is derived from the generalized variable description feature in the requirement knowledge block corresponding to $a$, while $V$ is derived from the generalized variable range feature.

(3) $\forall A \varsubsetneqq B, \exists r_{N T}: \varsubsetneqq \rightarrow R_{N T \rightarrow N T} E_{N T}^{A}, E_{N T}^{B}$. Then, $r_{N T}$ represents the mapping from $\varsubsetneqq$ to $R_{N T \rightarrow N T} \cdot E_{N T}^{A}$ and $E_{N T}^{B}$ represent the non-terminal entities corresponding to $A$ and $B$ respectively.

(4) $\forall a \in * R_{s}, \exists r_{T}: \varsubsetneqq \rightarrow R_{T \rightarrow N T} E_{T}^{a}, E_{N T}^{* R_{s}}$. Then, $r_{T}$ represents the mapping from $\in$ to $R_{T \rightarrow N T} . E_{T}^{a}$ represents the terminal entity corresponding to element $a$, while $E_{N T}^{* R_{s}}$ represents the non-terminal entity corresponding to set $* R_{s}$.

\section{A case study of mapping and classification of a simulated military aircraft horizontal tail control system requirement model}

This chapter takes a certain model of simulated military aircraft horizontal tail control system as an example. Based on the spreadsheet, some important product requirement information is mapped and classified through Python, and finally the graph database $\mathrm{Neo} 4 \mathrm{j}$ is used to display RMKG. $\mathrm{Neo} 4 \mathrm{j}$ is one of the most widely used graph databases at present. It stores structured data in the form of native graphs.

Referring to the internal data "Model Theory System of Product Life Cycle"[13], we obtain Input-Process-Output (IPO) diagram, as shown in Figure 2.

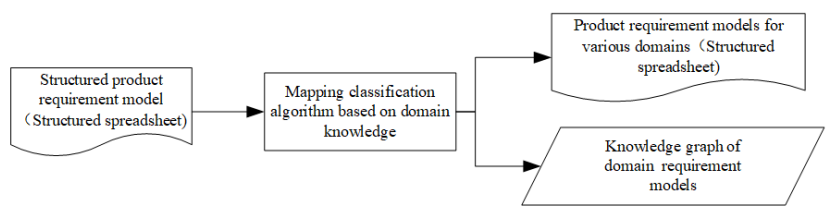

Figure 2 IPO diagram of product requirement model mapping and classification

Step 1 Using the structured and digital definition, RM is obtained from the technical agreement documents, and RMKG is shown in Figure 3.

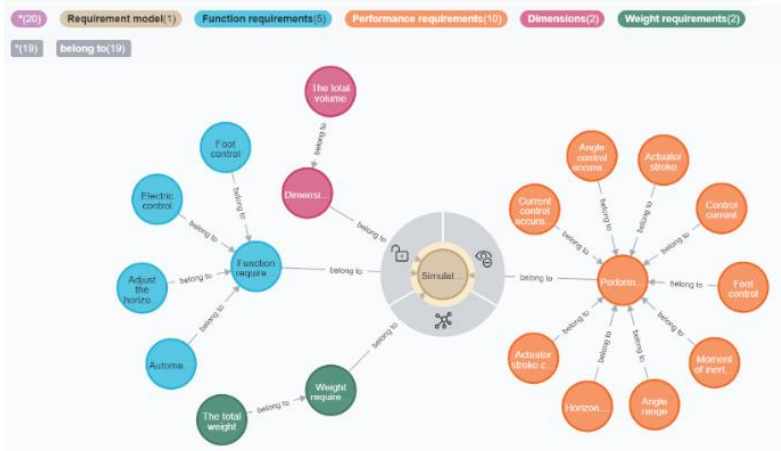

Figure 3 RMKG of the military aircraft horizontal tail control system

Step 2 Military aircraft horizontal tail control system involves professional knowledge of four domains: machinery, automatic control, fluid transmission, electrical and electronic. Based on the professional terms of the four domains, we established the domain knowledge bases and generated the knowledge graph of the domain knowledge bases. Figure 4 shows the knowledge base of the control domain. The knowledge of different domains is distinguished by different colors. There may be coupling between knowledge of different domains, such as the "sensitivity" in the figure, which belongs to both machinery and automatic control domains.

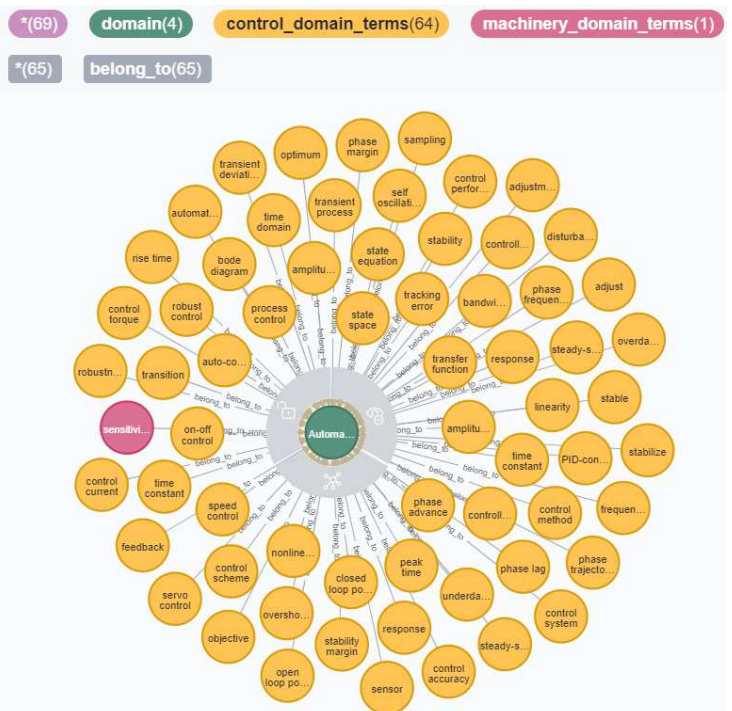

Figure 4 Domain knowledge base of the automatic control domain

Step 3 Taking RM as the input of the Python program. First, we added common requirement information such as product name, type description, price requirements and delivery time to the requirement model of each domain. Then we used jieba module to preprocess the remaining product requirement information. And then we calculated $\mathrm{Mr}$ 
between each word segmentation and each professional term by Eq. (5). After that, we used Eq. (6) to calculate the domain matching degree $\mathrm{DMr}$ between the requirement information and the domain knowledge base. Finally, according to the classification rules, the classification results of the requirement information are obtained. The main procedural steps of the classification rules are shown in Table 1.

Output the requirement form of each domain and the knowledge graph corresponding to the domain requirement models. The results are as follows, Table 2, Table 3, Table 4 and Table 5 are the tables of automatic control domain, the electrical and electronic domain, machinery domain and fluid transmission domain, respectively. Figure 5 shows the knowledge graph corresponding to the domain requirement models.
Table 1 Main procedural steps of classification rules

Algorithm Requirement mapping classification

/s represents a certain domain/

Is_model represents the corresponding domain requirement model/

1: for str in requirement_model $[* R]$

2: $\quad$ calculate DMr str, s by E.q(6)

3: $\quad S \leftarrow M H \leftarrow$

4: if Max $D M r>0$ then

$5:$

6:

$7:$

8 :

:

:

10:

11:

12 :

13 :

14 :

14:

15:

17 :

18: end for

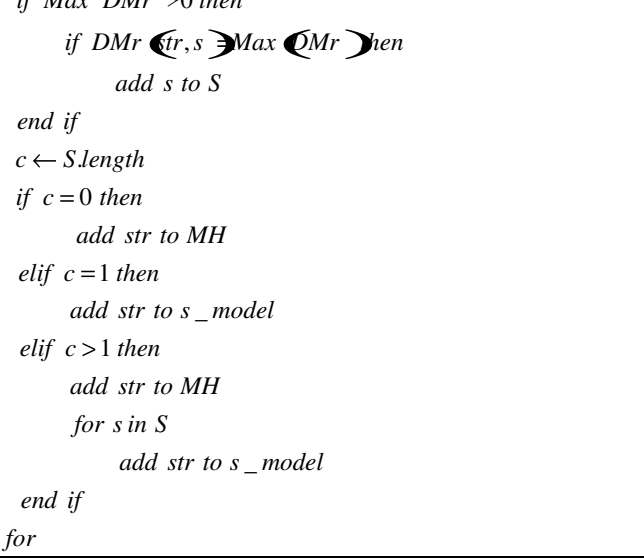

Table 2 Automatic control domain requirement table

\begin{tabular}{|c|c|c|c|c|c|}
\hline Product name & $\begin{array}{c}\text { Type } \\
\text { description }\end{array}$ & $\begin{array}{c}\text { Function } \\
\text { requirements }\end{array}$ & $\begin{array}{l}\text { Performance } \\
\text { requirements }\end{array}$ & price & Delivery time \\
\hline \multirow{4}{*}{$\begin{array}{c}\text { Fighter } \\
\text { Horizontal Tail } \\
\text { Control System }\end{array}$} & \multirow{4}{*}{ JXH-100 } & \multirow{2}{*}{$\begin{array}{l}\text { Adjust the horizontal tail } \\
\text { angle according to the } \\
\text { instruction }\end{array}$} & $\begin{array}{c}\text { Control current: } 4- \\
20 \mathrm{~mA}\end{array}$ & \multirow{4}{*}{$\begin{array}{l}\text { Less than } \\
\text { Three hundred } \\
\text { thousand } \\
\text { RMB }\end{array}$} & \multirow{4}{*}{90 days } \\
\hline & & & $\begin{array}{c}\text { Angle control accuracy: } \\
0.1^{\circ}\end{array}$ & & \\
\hline & & \multirow{2}{*}{ Automatic control } & $\begin{array}{c}\text { Current control } \\
\text { accuracy: } 0.05 \mathrm{~mA}\end{array}$ & & \\
\hline & & & $\begin{array}{l}\text { Actuator stroke control } \\
\text { accuracy: } 0.05 \mathrm{~cm}\end{array}$ & & \\
\hline
\end{tabular}

Table 3 Electrical and electronic domain requirement table

\begin{tabular}{|c|c|c|c|c|}
\hline Product name & $\begin{array}{c}\text { Type } \\
\text { description }\end{array}$ & $\begin{array}{l}\text { Performance } \\
\text { requirements }\end{array}$ & price & Delivery time \\
\hline \multirow{2}{*}{$\begin{array}{c}\text { Fighter } \\
\text { Horizontal Tail } \\
\text { Control System }\end{array}$} & \multirow{2}{*}{ JXH-100 } & $\begin{array}{c}\text { Control current: } 4- \\
20 \mathrm{~mA}\end{array}$ & \multirow{2}{*}{$\begin{array}{c}\text { Less than Three } \\
\text { hundred thousand } \\
\text { RMB }\end{array}$} & \multirow{2}{*}{90 days } \\
\hline & & $\begin{array}{l}\text { Current control } \\
\text { accuracy: } 0.05 \mathrm{~mA}\end{array}$ & & \\
\hline
\end{tabular}

Table 4 Mechanical domain requirement table

\begin{tabular}{|c|c|c|c|c|c|c|c|}
\hline Product name & $\begin{array}{c}\text { Type } \\
\text { description }\end{array}$ & Dimensions & $\begin{array}{c}\text { Weight } \\
\text { requirements }\end{array}$ & $\begin{array}{l}\text { Function } \\
\text { requirements }\end{array}$ & Performance requirements & price & $\begin{array}{l}\text { Delivery } \\
\text { time }\end{array}$ \\
\hline \multirow{6}{*}{$\begin{array}{c}\text { Fighter } \\
\text { Horizontal } \\
\text { Tail Control } \\
\text { System }\end{array}$} & \multirow{6}{*}{ JXH-100 } & \multirow{6}{*}{$\begin{array}{l}\text { The total volume } \\
\text { is less than } \\
1500 \times 80 \times 20\end{array}$} & \multirow{6}{*}{$\begin{array}{l}\text { The total weight } \\
\text { is less than } 300 \mathrm{~kg}\end{array}$} & \multirow{3}{*}{ Foot control } & Angle range: $-25^{\circ} \sim 25^{\circ}$ & \multirow{6}{*}{$\begin{array}{l}\text { Less than Three hundred } \\
\text { thousand RMB }\end{array}$} & \multirow{6}{*}{90 days } \\
\hline & & & & & $\begin{array}{l}\text { Moment of inertia: } \\
6.25 \mathrm{~kg} \cdot \mathrm{m}^{2}\end{array}$ & & \\
\hline & & & & & Foot control: $0 \sim 10^{\circ}$ & & \\
\hline & & & & \multirow{3}{*}{$\begin{array}{l}\text { Electric } \\
\text { control }\end{array}$} & Actuator stroke: $20 \mathrm{~cm}$ & & \\
\hline & & & & & $\begin{array}{l}\text { Actuator stroke control } \\
\text { accuracy: } 0.05 \mathrm{~cm}\end{array}$ & & \\
\hline & & & & & Horizontal tail load: $300 \mathrm{kN}$ & & \\
\hline
\end{tabular}


Table 5 Fluid transmission domain requirement table

\begin{tabular}{|c|c|c|c|c|}
\hline Product name & $\begin{array}{c}\text { Type } \\
\text { description }\end{array}$ & $\begin{array}{l}\text { Performance } \\
\text { requirements }\end{array}$ & price & Delivery time \\
\hline \multirow{2}{*}{$\begin{array}{c}\text { Fighter } \\
\text { Horizontal Tail } \\
\text { Control System }\end{array}$} & \multirow[b]{2}{*}{ JXH-100 } & Actuator stroke: $20 \mathrm{~cm}$ & \multirow{2}{*}{$\begin{array}{c}\text { Less than Three } \\
\text { hundred thousand } \\
\text { RMB }\end{array}$} & \multirow[b]{2}{*}{90 days } \\
\hline & & $\begin{array}{c}\text { Actuator stroke control } \\
\text { accuracy: } 0.05 \mathrm{~cm}\end{array}$ & & \\
\hline
\end{tabular}

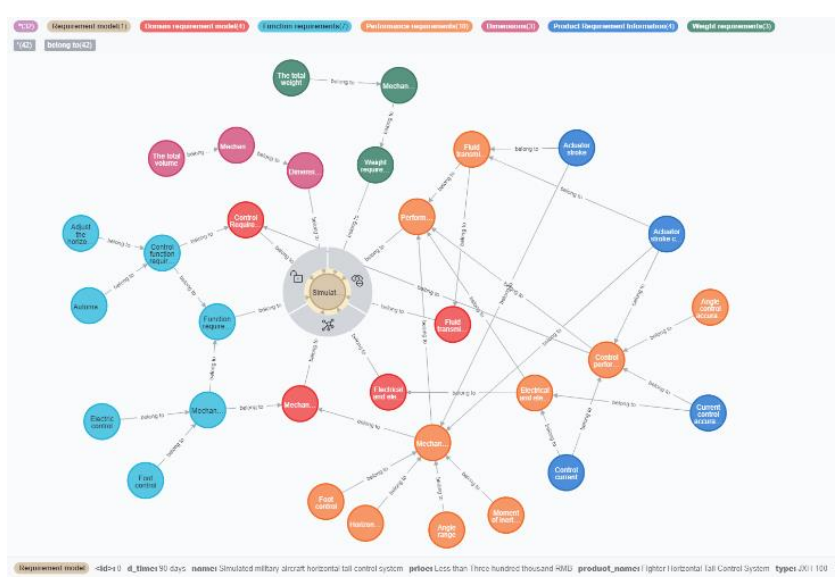

Figure 5 knowledge graph of the domain requirement models

It can be seen from the mapping results that different entity types are distinguished by different colors. If the requirement information is confused, that is, the requirement information exists in multiple domain requirement models at the same time, when mapping it to the terminal ontology, the type is "product requirement information".

(1) Both the control requirement model and the electrical and electronic requirement model include Control current and Control current accuracy.

(2) Both the mechanical requirement model and the fluid transmission requirement model include Actuator stroke.

(3) The control requirement model, the mechanical requirement model and the fluid transmission requirement model all contain Actuator stroke control accuracy.
The confusing requirement information is finally output to the product developer. This information may be further divided, or it may be determined by the product developer whether this information needs to be output to multiple departments at the same time. For the latter, it means that the completion of these indicators requires the interdisciplinary collaboration of designers from multiple departments.

For the requirement information that is not confusing, when it is mapped to the terminal ontology, the type inherits the type of its parent entity.

\section{Product requirement model management based on Hoteam PLM8.0 platform}

In the current PLM project management, project-related information stored in the project folder is still based on unstructured documents. Translating this information into models through relevant model definition methods and using the model as the smallest unit of system management are the keys to upgrading PLM to PLMM. Based on the Hoteam PLM8.0 platform, we provided a preliminary model-based efficient management framework for the product requirement model. It can provide a basis for product model collaboration and PLMM research and development. The specific management architecture and display path are respectively shown in Figure 6 and Figure 7. 


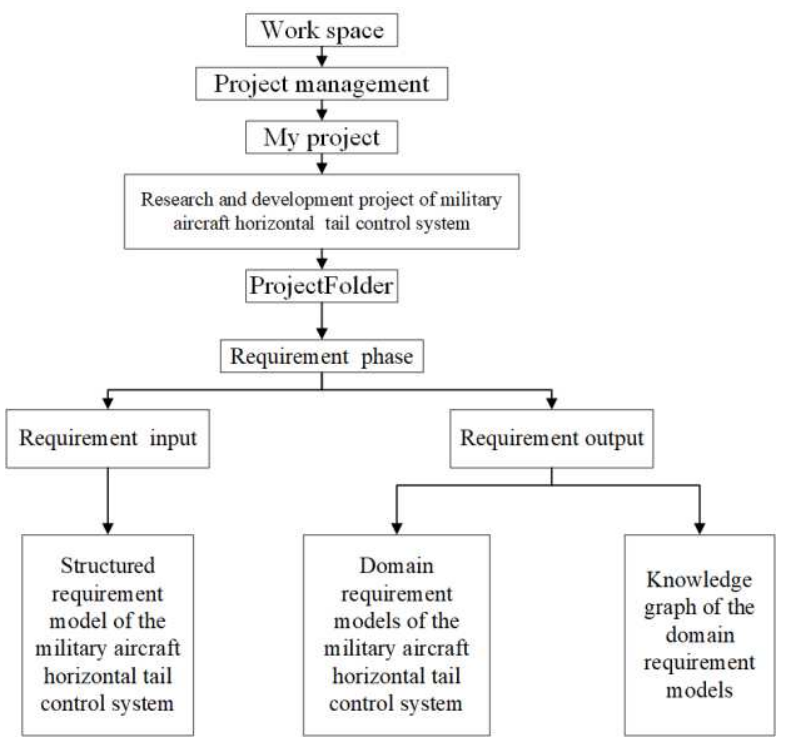

Figure 6 management architecture
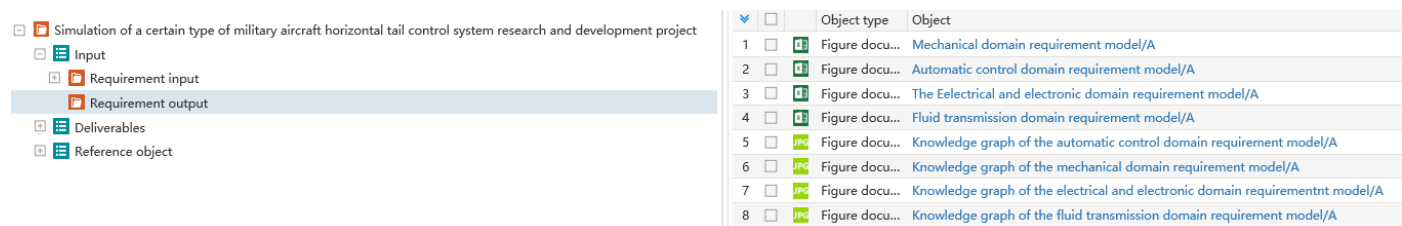

Figure 7 display path of the product requirement models

Requirements are the important bases for production. According to the important requirement information, the product manufacturer will strictly approve it and make feasibility demonstration. However, most manufacturing enterprises need to meet for discussion currently. This collaborative mode of centralized meeting cannot meet the needs of operation in different places, that is, when different departments are in different places, they cannot carry out collaborative approval work in a timely manner. To improve the agility of collaboration among departments, based on the efficient management of the model, the process approval function of PLMM can be used to realize cross-regional collaboration among departments.

\section{Conclusions}

The main contributions are summarized as follows.

(1) Aiming at the existing unstructured product requirement information, the structured and digital definition is used to transform it into a product requirement model.

(2) A requirement model mapping and classification method based on domain knowledge for mechatronic products is proposed. Taking a simulated military aircraft horizontal tail control system as an example, this paper realized the automatic generation of requirement models of various domains, and displayed the relationship between each requirement information and requirement model through knowledge graph.

(3) Based on PLM8.0 platform, a development framework to realize efficient management of product requirement model is presented.

(4) This paper provides a basis for the research and development of model collaboration technology and PLMM system, and provides technical support for the improvement of product digitization rate and collaborative agility.

\section{Declaration}

\section{Acknowledgements}

The authors sincerely thanks to Associate Professor Yanchao Dong of Tongji University for his critical discussion and reading during manuscript preparation.

\section{Funding}

This work was supported by key research and 
development project of China (Grant No. 2018YFB1700900).

\section{Availability of data and materials \\ Not applicable}

\section{Authors' contributions}

The author' contributions are as follows: JY and FW was in charge of the whole trial; FW wrote the manuscript; $\mathrm{ZC}$ and $\mathrm{HZ}$ helped perfect the mapping and classification method with constructive discussions. JL and PW provided Hoteam PLM8.0 platform for analyses and model management. All authors read and approved the final manuscript.

\section{Competing interests}

The authors declare no competing financial interests.

\section{Consent for publication}

Not applicable

\section{Ethics approval and consent to participate}

Not applicable

\section{References}

[1] C Semeraro, M Lezoche, H Panetto, et al. (2019). Data-Driven Pattern-Based Constructs Definition for the Digital Transformation Modelling of Collaborative Networked Manufacturing Enterprises. IFIP Advances in Information and Communication Technology. doi: 10.1007/978-3-030-28464-0 44.

[2] Berenbach, Brian A. (2004). Comparison of UML and text based requirements engineering. Association for Computing Machinery. doi: $10.1145 / 1028664.1028766$.

[3] M Ballard, R Peak, S Cimtalay \& D Mavri. Bidirectional Text-toModel Element Requirement Transformation. IEEE Aerospace Conference, Big Sky, MT, USA, 2020, pp. 1-14. doi: 10.1109/AERO47225.2020.9172306.

[4] M Mayda, H R Borklu. A Computational Framework for ModelBased Innovative Conceptual Design Process. Journal of the Chinese Society of Mechanical Engineers, 2016, 37(1), 55-60.

[5] B Cuesta (2018). Model-based approach for agile requirements engineering using SysML and Papyrus. 5th International Meeting of Technological Innovation, Ocana, Colombia. doi: 10.1088/17426596/1257/1/012013.

[6] Jihong Liu, Shude Wang, Chao Fu. Design Analysis Method for Multidisciplinary Complex Product using SysML. MATEC Web of Conferences,2017,139. doi: 10.1051/matecconf/201713900014.

[7] Xu Zihe. Development of MBSE-oriented complex product requirement modeling and tracking system [D]. Zhejiang University, 2017. (in Chinese)

[8] Luo X., Zhou Y., Liu B., Wang Y. (2016). Research on OntologyBased Knowledge Modeling of Design for Complex Product. Computational Intelligence and Intelligent Systems. Communications in Computer and Information Science, 2016, 575: 665-671.
[9] Yin Xuemei, Zhou Junhua, Zhu Yaoqin. Data modeling and datadriven method in collaborative design of complex products. Journal of Computer Applications. 2018, 38(10).

[10]O Yaman, B Zhu, U Roy. Towards the development of an ontologybased product requirement model. ASME 2014 International Mechanical Engineering Congress and Exposition. Montreal, Quebec, Canada. doi: 10.1115/IMECE2014-38693.

[11]M Bakhshandeh, G Antunes, R Mayer. A modular ontology for the enterprise architecture domain. 2013 17th IEEE International Enterprise Distributed Object Computing Conference Workshops. IEEE, Vancouver, BC, Sept 9-13, 2013: 5-12.

[12]Zhongqi Sheng, Yazi Wang, Junyou Song. Customer requirement modeling and mapping of numerical control machine. Advances in Mechanical Engineering, 2015, 7(10).

[13]Liu Yusheng. Model Theory System of Product Life Cycle [R], Zhejiang: Zhejiang University CAD\&CG State Key Laboratory, 2020. (in Chinese)

[14]Chen Z., Wang Y., Zhao B., Cheng J., et al. Knowledge Graph Completion: A Review. IEEE Access, vol. 8, pp. 192435-192456, 2020, doi: 10.1109/ACCESS.2020.3030076.

[15]X Zou (2020). A Survey on Application of Knowledge Graph. Journal of Physics: Conference Series. doi: 10.1088/17426596/1487/1/012016.

[16]J Papinniemi, L Hannola, M Maletz. Challenges in integrating requirements management with PLM. International Journal of Production Research, 2014 , 52(15): 4412-4423, doi: 10.1080/00207543.2013.849011.

[17]S Maltzahn, R Anderl. Early BOM Derivation From Requirement Specifications by Reusing Product Knowledge. Proceedings of the ASME 2011 International Design Engineering Technical Conferences and Computers and Information in Engineering Conference. 2011. pp. 1189-1195.

[18]Xiaojie Ma, Guofu Ding, Shengfeng Qin, et al. Transforming Multidisciplinary Customer Requirements to Product Design Specifications. Chinese Journal of Mechanical Engineering, 2017, 30 (5): 1069-1080.

[19]G Bruno, D Antonelli, A Villa. A Reference Ontology to Support Product Lifecycle Management, Procedia CIRP, 2015: 41-46.

[20]Jian Cui, Guoning Qi, Yangjian Ji, et al. Management model on requirement information oriented to product lifecycle. Computer Integrated Manufacturing Systems, CIMS, 2007, 13(12): 2406-2414.

[21]B Chabot, P Gautreau, B Sommacal. How to Improve PLM Approach Efficiency Based on Knowledge Engineering, Knowledge Management and Semantic Web Technologies Domains? IFIP Advances in Information and Communication Technology, 2014, vol 442

[22] Guangzhong Hu, Xinjian Xu, Shoune Xiao, et al. Product Data Model for Performance-driven Design. Chinese Journal of Mechanical Engineering, 2017, 30(5): 1112-1122.

[23]J Guia, V G Soares, J Bernardino. Graph databases: Neo4j analysis. 19th International Conference on Enterprise Information Systems. April 26-29, 2017.

[24]Jiang Dianxiang, Liu Zhuo, Lin Ziping. Measurement of the moment of inertia of a certain type of plane horizontal tail [C]. Proceedings of the 12th Academic Exchange Conference of the Weight Engineering Professional Committee of the Chinese Aeronautical Society. 2008: 33-41. (in Chinese)

[25]Yue Cao, Jing Xu, Yusheng Liu, Xiaoping Ye, Jianjun Zhao. Automated generation of control logic from system design based on SysML and the IEC 61499 Function Block. Proceedings of the 
Institution of Mechanical Engineers, Part B: Journal of Engineering Manufacture, 2019, 233(14).

[26]C Wang. MBSE-Compliant Product Lifecycle Model Management. 2019 14th Annual Conference System of Systems Engineering (SoSE), Anchorage, AK, USA, 2019, pp. 248-253, doi: 10.1109/SYSOSE.2019.8753869.

\section{Biographical notes}

Jiguang Yue, born in 1961, is currently a professor at School of Electronic and Information Engineering, Tongji University. He received his $\mathrm{Ph} . \mathrm{D}$. degree in fluid transmission and control from Harbin Institute of Technology. His research interests include advanced measurement and control theory research and technology development in the field of electromechanical integration.

E-mail: yuejiguang@tongji.edu.cn

Fuchao Wu, born in 1998, is currently a master candidate at School of Electronic and Information Engineering, Tongji University. His research interests include product lifecycle model management for complex mechatronic products.

Tel: 18907852667; E-mail: 295035544@qq.com

Jun Liu, born in 1980, is currently the president at Shandong Hoteam software Co., Ltd, China. His research interests include intelligent manufacturing and digital design process manufacturing. E-mail: liujun@hoteamsoft.com

Ping Wang, born in 1975, is currently the managing director at Shandong Hoteam software Co., Ltd, China. Her research interests include intelligent manufacturing, design knowledge and process management.

E-mail: wpdzxx@126.com

Zhexin Cui, born in 1998, is currently a master candidate at School of Electronic and Information Engineering, Tongji University. His research interests include product lifecycle model management for complex mechatronic products.

E-mail:932529361@qq.com

Hongyu Zou, born in 1997, is currently a master candidate at School of Electronic and Information Engineering, Tongji University. His research interests include groupware technology.

E-mail: 2030754@tongji.edu.cn

\section{Appendix}

Not applicable 


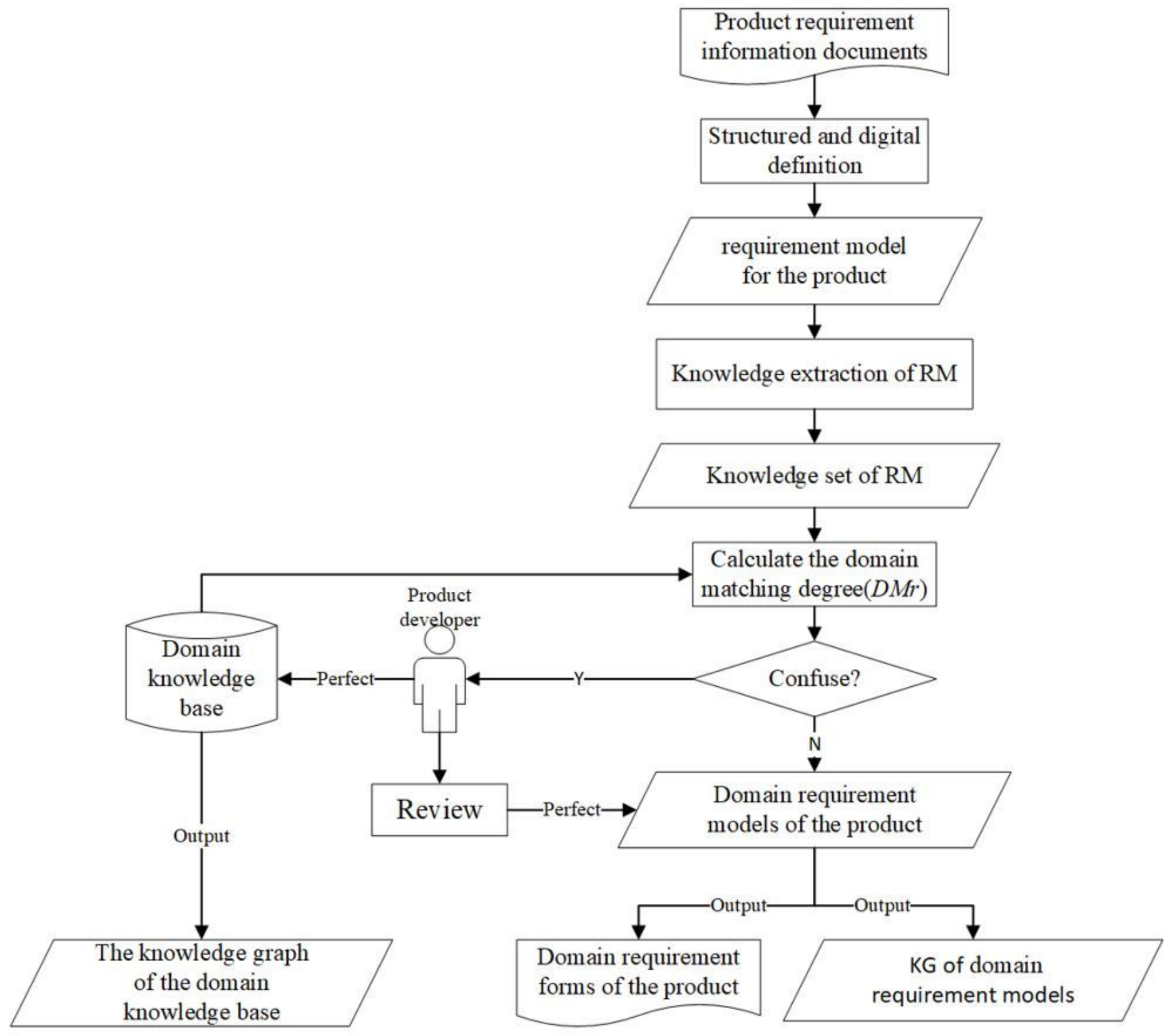

Figure 1

mapping and classification mechanism of RM based on domain knowledge 


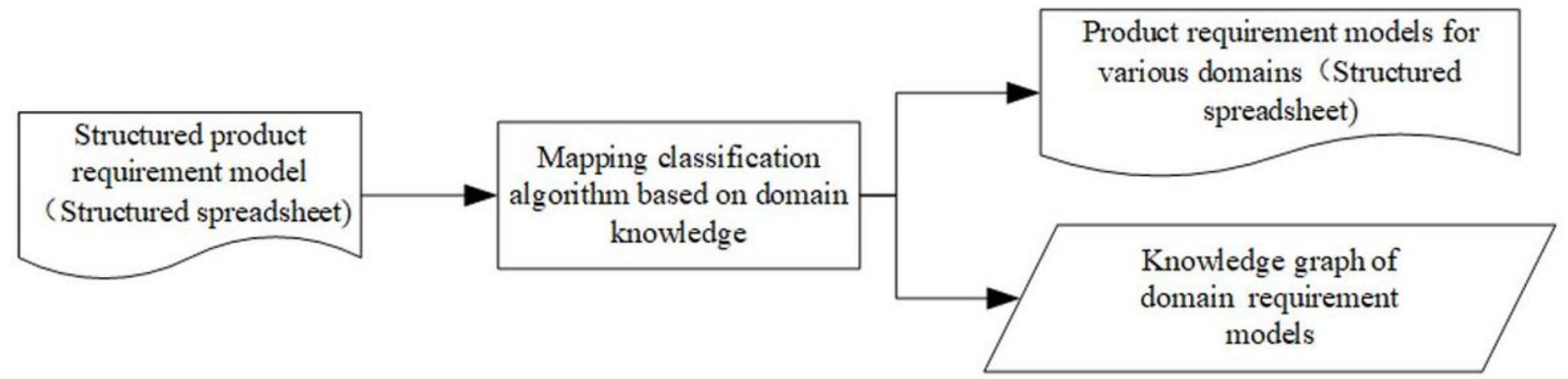

\section{Figure 2}

IPO diagram of product requirement model mapping and classification

(20) Requirement model (1)

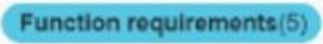

Pertormance requirements(10)

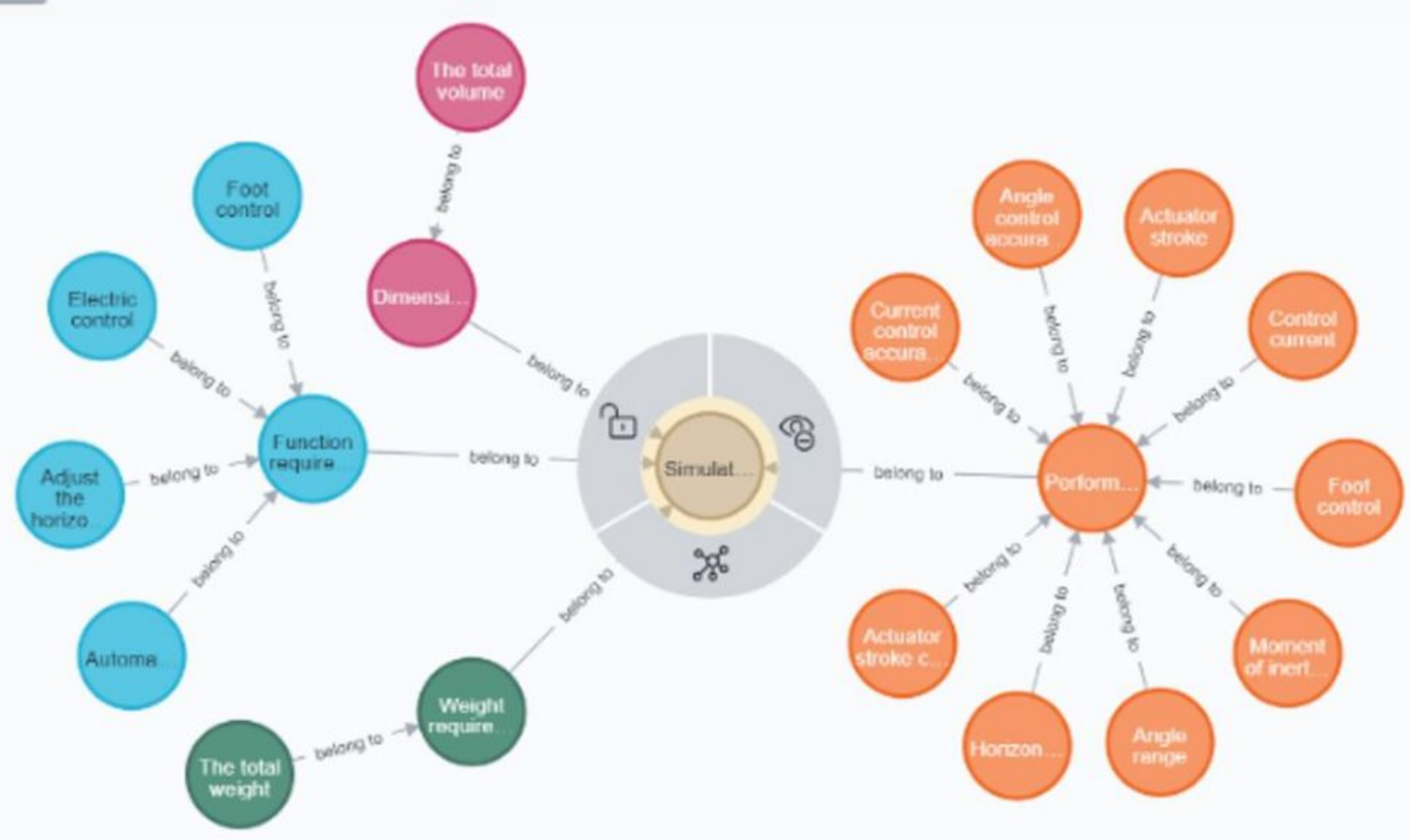

\section{Figure 3}

RMKG of the military aircraft horizontal tail control system 


\section{*(69) domain(4) control_domain_terms(64) machinery_domain_terms(1)}

\section{*(65) belong_to(65)}

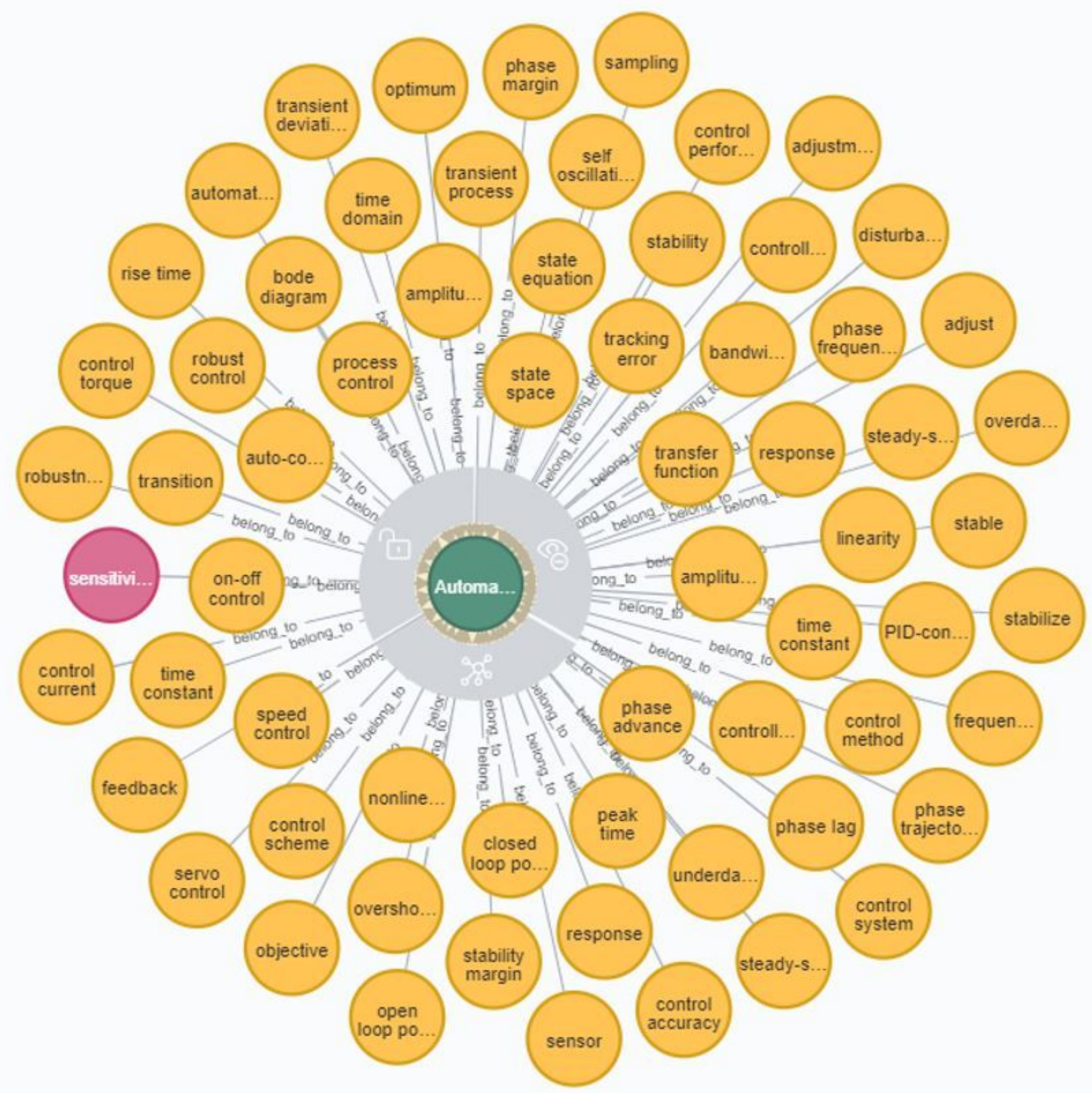

Figure 4

Domain knowledge base of the automatic control domain 


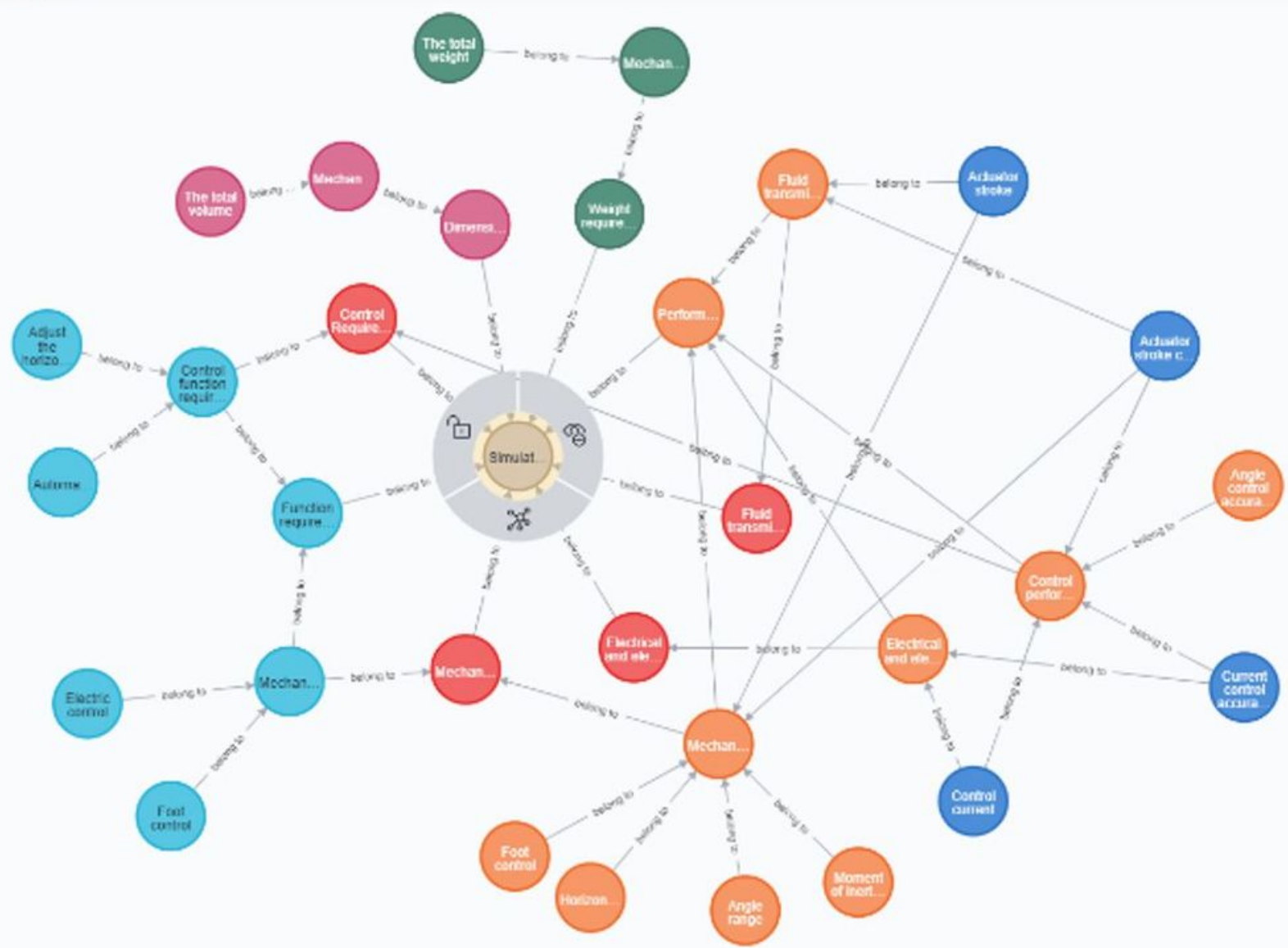

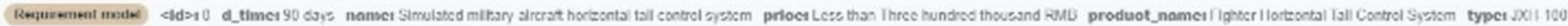

\section{Figure 5}

knowledge graph of the domain requirement models 


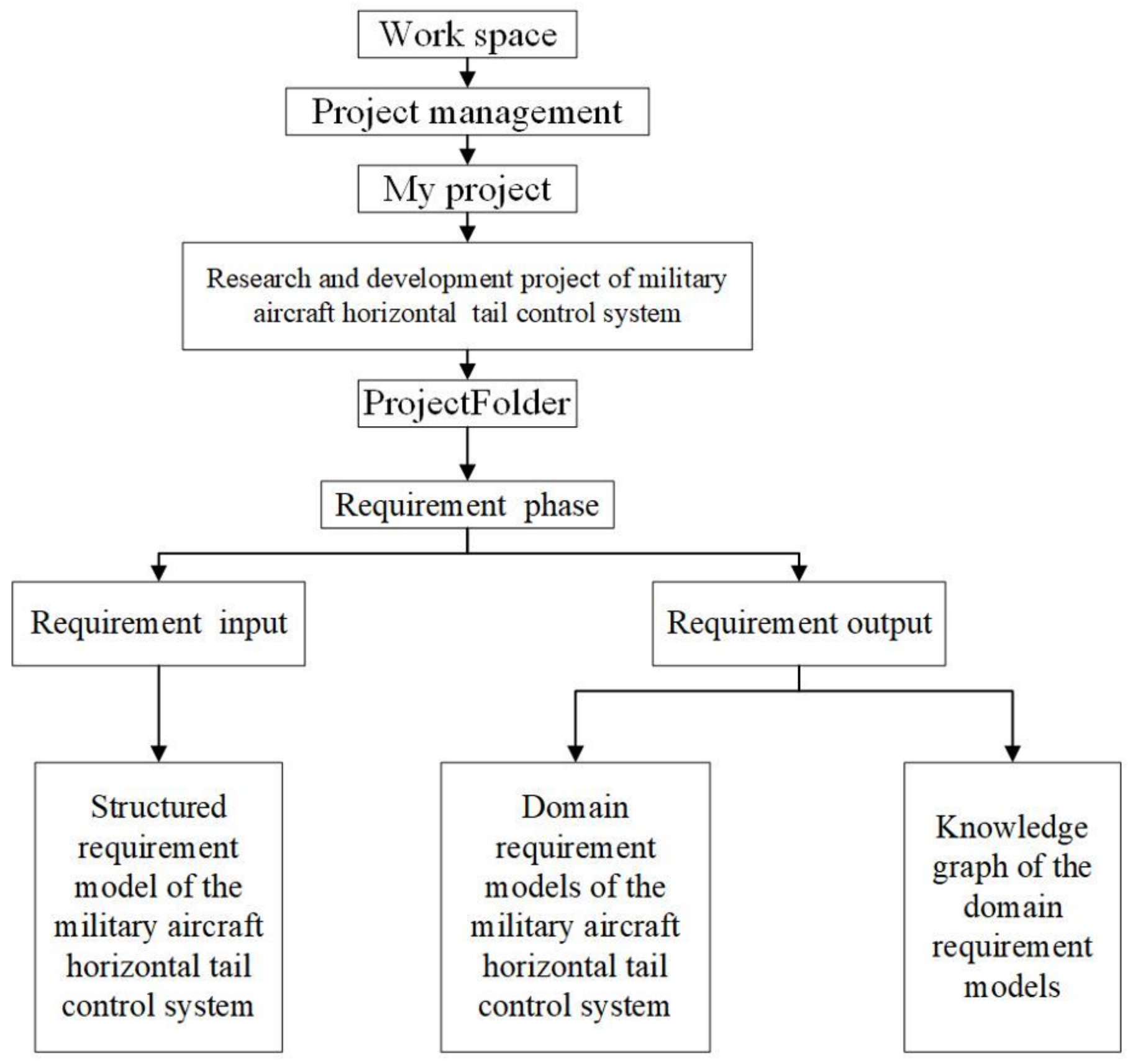

Figure 6

management architecture

$\square$ Simulation of a certain type of military aircraft horizontal tail control system research and development project
$\square$ 目 Input
QRequirement input
$\square$ Requirement output
$\square$ Deliverables
$\square$ Reference object

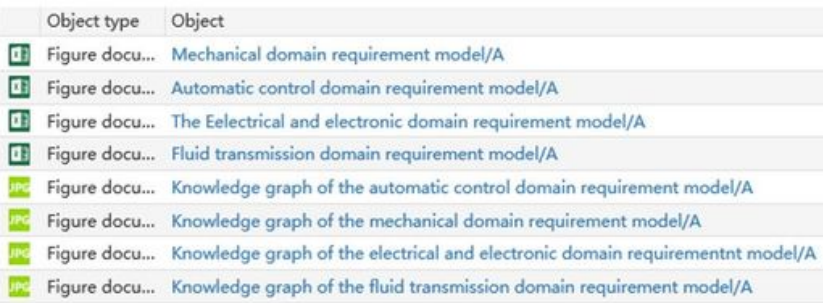


display path of the product requirement models 